\title{
In COVID-19 pneumonia, high-sensitive troponin I independently related with tricuspid regurgitation pressure gradient
}

\author{
Akkan Avci ${ }^{1}$, Hilmi Erdem Sumbul ${ }^{1}$, Yahya Kemal Icen ${ }^{1}$, Abdullah Orhan Demirtas ${ }^{1}$, \\ Huseyin Ali Ozturk ${ }^{1}$, Arzu Pinar ${ }^{1}$, Edip Bayrak ${ }^{1}$, Mehmet Uzucek ${ }^{1}$, Begum Seyda Avci ${ }^{1}$, \\ Onder Yesiloglu ${ }^{1}$, Salim Satar ${ }^{1}$, Hayri Cinar ${ }^{1}$, Hasan Koca ${ }^{1}$, Burcak Cakir Pekoz ${ }^{1}$, Tuba \\ Turunc $^{1}$, Ilker Unal ${ }^{2}$, Murat Erdogan ${ }^{1}$, and Mevlut Koc ${ }^{1}$ \\ ${ }^{1}$ Health Science University, Adana City Research and Training Hospital \\ ${ }^{2}$ Cukurova University Faculty of Medicine
}

October 24, 2020

\begin{abstract}
Background: We aimed to determine the clinical, laboratory and echocardiographic parameters associated with an increased high-sensitivity cTnI (hs-cTnI) levels in COVID-19 pneumonia who admitted to the emergency department. Methods: This cross-sectional study included 70 COVID-19 patients with and without pneumonia. In addition to medical history, physical examination and laboratory tests, echocardiography and lung computed tomography was performed. The hs-cTnI value of the patients' [?]14ng/L for men and [?]18ng/L for women was considered as an increased hs-cTnI. The patients were compared in two groups, with and without pneumonia. Results: Tricuspid regurgitation pressure gradient (TRPG) were higher in patients with COVID-19 pneumonia $(\mathrm{p}<0.05)$. Tricuspid annular plane systolic excursion (TAPSE) were lower in patients with COVID19 pneumonia $(\mathrm{p}<0.05)$. In regression analysis; HR, NT-proBNP and TRPG was found to be related to hs-cTnI $(\mathrm{p}<0.01)$. Discussion: The most important determinants of increased hs-cTnI level in these patients are; increased NT-proBNP and TRPG. Therefore, it is necessary to follow-up the RV functions and TRPG by echocardiography in patients with increased hs-cTnI and COVID-19 pneumonia.
\end{abstract}

\section{Hosted file}

Main_manuscript.pdf available at https://authorea.com/users/369858/articles/488667-incovid-19-pneumonia-high-sensitive-troponin-i-independently-related-with-tricuspidregurgitation-pressure-gradient 


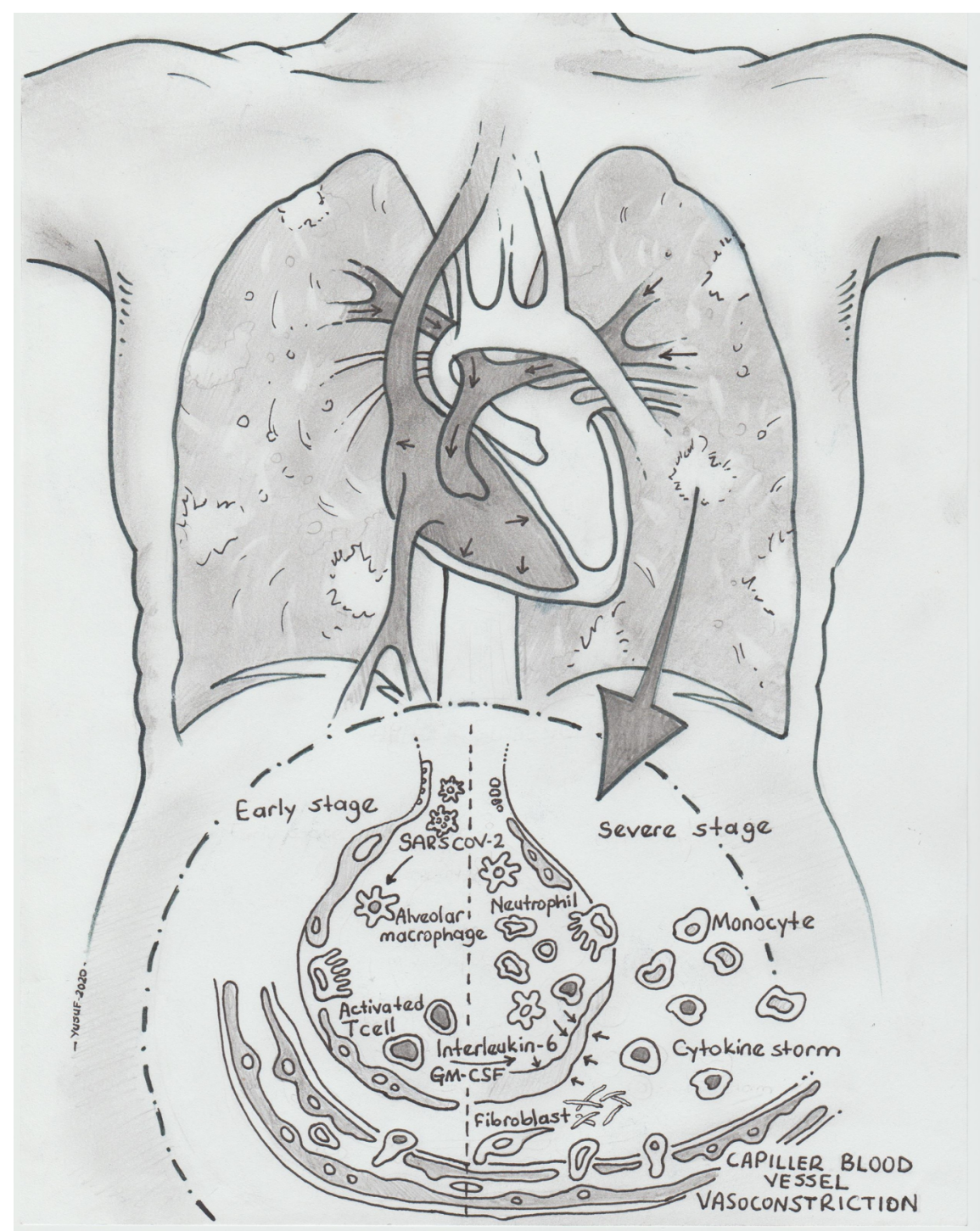



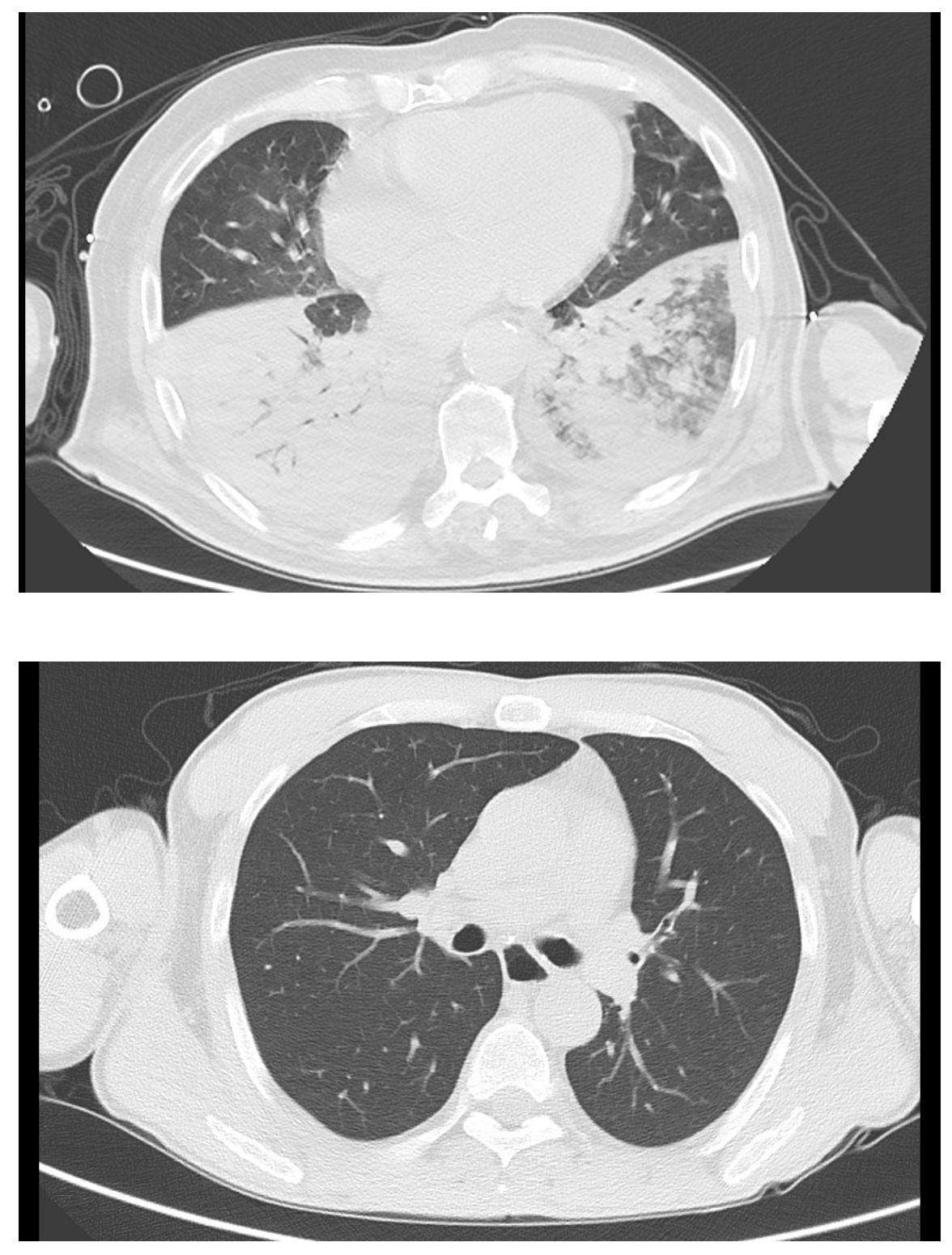


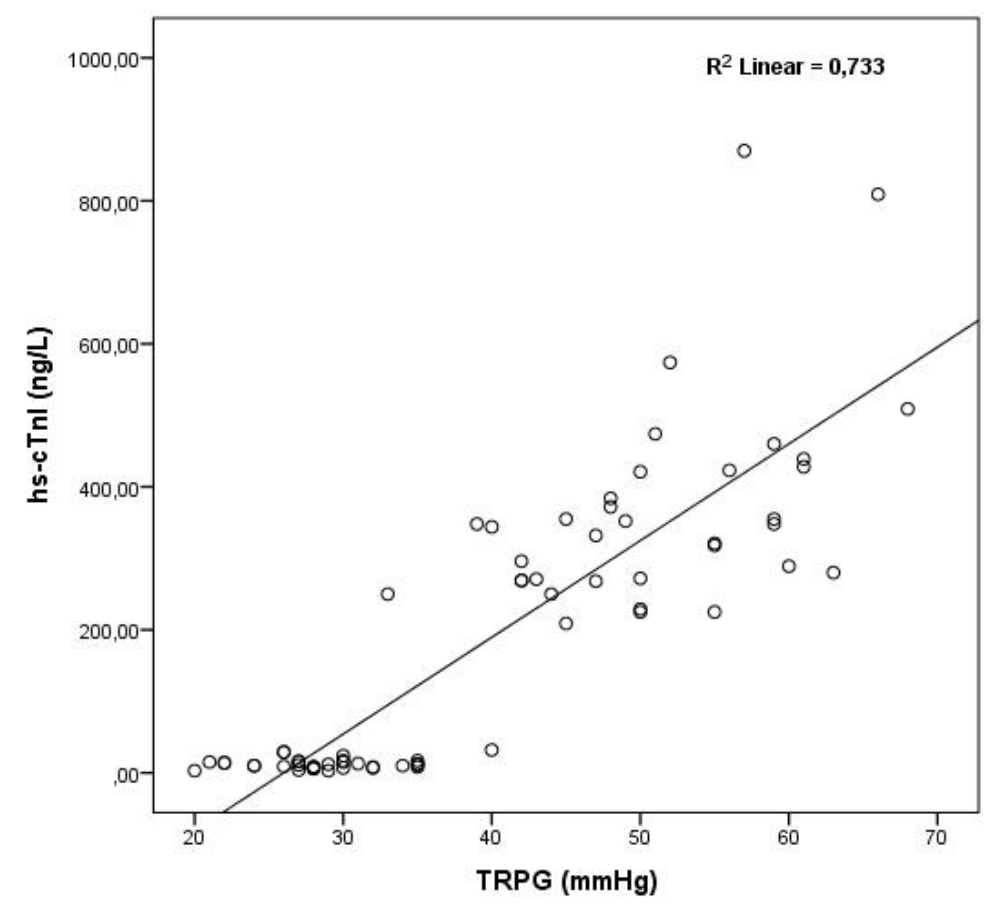

